\title{
Shear stress and aneurysms: a review
}

\author{
Brittany Staarmann, MD, ${ }^{1}$ Matthew Smith, MD, ${ }^{2}$ and Charles J. Prestigiacomo, MD ${ }^{1}$ \\ Departments of ${ }^{1}$ Neurosurgery and ${ }^{2}$ Neurology, University of Cincinnati Medical Center, Cincinnati, Ohio
}

\begin{abstract}
Wall shear stress, the frictional force of blood flow tangential to an artery lumen, has been demonstrated in multiple studies to influence aneurysm formation and risk of rupture. In this article, the authors review the ways in which shear stress may influence aneurysm growth and rupture through changes in the vessel wall endothelial cells, smooth-muscle cells, and surrounding adventitia, and they discuss shear stress-induced pathways through which these changes occur. https://thejns.org/doi/abs/10.3171/2019.4.FOCUS19225
\end{abstract}

KEYWORDS shear stress; aneurysm; histology; vessel wall

$\mathrm{H}$ ow the hemodynamic factor of wall shear stress (WSS) affects an aneurysm's natural history is the subject of debate. Both high and low WSS are implicated in aneurysmal growth, and aneurysm wall tissue may encounter both high and low WSS, yet, only a minority of aneurysms rupture. Therefore, factors intrinsic to the vessel wall's reaction to the changing stresses placed upon it must influence the risk of rupture. In this article, we discuss how hemodynamic forces, namely WSS, encountered by the aneurysm wall relate to tissue changes in the wall, and how these tissue changes relate to aneurysmal growth and rupture.

\section{Methods}

A comprehensive review of the literature was performed using a search of PubMed, EBSCO, and Scopus with the following keywords: WSS, inflammation, histological, and hemodynamic, coupled with aneurysm. Relevant articles were selected based on individual merit and included basic science research, case series, human subjects research, reviews, and book chapters. Further sources were obtained from the references of selected articles.

\section{Normal and Aneurysmal Vascular Structure}

There are three layers within the vessel wall: the intima, the media, and the adventitia. The innermost layer, the intima, is composed of a single layer of endothelial cells (ECs) on the luminal side of the vessel and is in direct contact with blood flow. The media contains concentric sheets of smooth-muscle cells (SMCs) and collagen (pre- dominately type III). The SMCs help generate and regulate the production of the extracellular matrix (ECM), consisting of elastin, collagen, proteoglycans, and fibrillin..$^{20}$ Of note, at vessel bifurcations, it has been demonstrated that the apical portions of intracranial vessels lack SMCs (a gap referred to as the "medial raphe"). ${ }^{44}$ The adventitia, the outer layer, consists of type I collagen, elastin, fibroblasts, nerves, and the vasa vasorum. The internal elastic lamina (IEL) is a membrane of elastic fibers that separates the intima from the media. The external elastic lamina, present in most arteries and separating the adventitia from the media, is not present in the intracranial arteries. ${ }^{35}$

The aneurysm wall structure demonstrates changes in the histology of the vessel wall: a loss of IEL, thinning of the tunica media, and degeneration of the ECM. ${ }^{28}$ The fragility of the damaged vascular wall is related to the risk of rupture. ${ }^{22}$ The aneurysm wall histology has been categorized by Frösen et al. to include four subtypes which were then correlated to risk of rupture: 1) endothelialized wall with linearly organized SMCs (42\% ruptured); 2) thickened wall with disorganized SMCs (55\% ruptured); 3 ) hypocellular wall with either myointimal hyperplasia or organizing luminal thrombosis (64\% ruptured); and 4) extremely thin thrombosis-lined hypocellular wall (100\% ruptured).$^{13}$ If aneurysm walls can have both increased thickness and cell proliferation, as well as decreased thickness and hypocellularity, then multiple processes may influence the progression from normal vasculature to ruptured aneurysm. Vessel-wall enhancement has been shown to predict aneurysm instability, while nonenhancement predicts stability ${ }^{41}$ therefore, changes in the aneurysm wall itself may contribute to the risk of rupture.

ABBREVIATIONS EC = endothelial cell; ECM = extracellular matrix; IEL = internal elastic lamina; $\mathrm{MMP}=$ matrix metalloproteinase; $\mathrm{NO}=$ nitric oxide; $\mathrm{SMC}=$ smoothmuscle cell; tPA = tissue plasminogen activator; WSS = wall shear stress; WSSG = WSS gradient.

SUBMITTED March 1, 2019. ACCEPTED April 8, 2019.

INCLUDE WHEN CITING DOI: 10.3171/2019.4.FOCUS19225. 
How a normal vessel wall is transformed into an aneurysm is multifactorial, but changes at the cellular level initiated by mechanical stimuli, such as WSS, appear to play a critical role in the progression of an aneurysm. In the pages that follow, we will discuss the histological changes found in aneurysms correlated with shear stress and how these changes may predispose to aneurysm growth and rupture.

\section{WSS: An Overview}

WSS is a frictional force from blood flow tangential to the arterial lumen. Endothelial function is regulated by normal (1.5-2.5 Pa) WSS. ${ }^{9}$ Wall pressure is the kinetic energy of blood transferred as inertial force perpendicular to the vessel surface, and wall tension is the force exerted by the wall in equal magnitude and opposite direction to the wall pressure. ${ }^{30}$ When the wall tension required is greater than the tensile strength of the vessel wall, the aneurysm ruptures. WSS can increase and decrease depending on the velocity and viscosity of blood. ${ }^{30} \mathrm{~A}$ gradient in the degree of WSS (WSS gradient [WSSG]) is then created as blood flow velocity increases or decreases, depending on the flow patterns in the vessel. Accelerating flow creates a positive WSSG, while decelerating flow creates a negative WSSG.

The flow patterns experienced by aneurysm wall tissue are complex and may change throughout the course of an aneurysm's history: high WSS experienced during initiation of aneurysm blebs may then proceed to low WSS as the geometry of the aneurysm changes. ${ }^{28}$ Both high and low WSS have been associated with aneurysm growth and rupture. Bleb formation has been found at or adjacent to zones of increased WSS, as opposed to zones of stagnation. ${ }^{6}$ In a study by Jou et al., mean WSS for ruptured and unruptured aneurysms was not statistically different as assessed by computational flow dynamics. However, there was a statistically significant difference in the ruptured and unruptured aneurysms in the area of aneurysm wall encountering low WSS, with a greater area being exposed to low WSS in the ruptured than unruptured aneurysms. ${ }^{19}$ Similar computational flow dynamic studies by Xiang et al. did show an association between low WSS and rupture. ${ }^{45}$ In addition, Boussel et al. demonstrated aneurysm growth to be more likely to occur in regions where the endothelium is exposed to low WSS. ${ }^{4}$ Thin-walled regions of the aneurysm dome have been colocalized with areas of low wall stress in unruptured cerebral aneurysms. ${ }^{21}$ Furthermore, Cebral et al. demonstrated that flow characteristics in hyperplastic regions of aneurysms are similar to those found in atherosclerotic plaques, with low WSS in regions of slow or recirculating flow. ${ }^{5}$ The changes in shear stress may also play a role in vessel wall transformation: Doddasomayajula et al. have demonstrated using ipsilateral and mirrored aneurysms in patients with ruptured aneurysms (to avoid confounding additional patient variables) that high WSS oscillations are associated with rupture. These ruptured aneurysms were found to have greater maximal and lower minimal shear stress compared to their unruptured counterparts. ${ }^{8}$ The gradient of the WSS has been found to have an effect on cells of the vascular wall, as Dolan et al. have demonstrated that a negative WSSG may antagonize the effect of high WSS on gene expression. ${ }^{10}$ An additional theory by Meng et al. is that both high and low WSS drive aneurysm growth and rupture, with regions of low WSS with high oscillatory shear index undergoing inflammatory cell-mediated destructive remodeling, and regions of high WSS with a positive WSSG undergoing mural cell-mediated remodeling. ${ }^{28}$

\section{Shear Stress and Endothelial Changes}

Injury to the endothelium is presumed to be one of the initiating factors in aneurysm formation. ECs, directly in contact with blood flow under normal conditions, are sensitive to WSS and require it for normal function. Mechanoreceptors of WSS are located on the apical and basal surfaces of ECs and include ion channels, integrins, cell adhesion molecules, and G-protein-coupled receptors and can lead to prolonged activation of inflammatory pathways such as NF-xB. ${ }^{43}$ Abnormalities of the WSS may cause dysfunction and/or loss of the endothelial layer. Additionally, ECs help prevent thrombus formation on the vessel wall; however, when loss of WSS due to thrombus development does occur, there is dysregulation of the associated ECs. ${ }^{25,38}$

Shear stress is considered an important driver of ECs in the development of new blood vessels. ${ }^{25}$ Laminar shear stress on the EC promotes arrest of the cell cycle in $\mathrm{G}_{1}$ or $\mathrm{G}_{0}$. However disturbed shear stress accelerates EC turnover, and low shear stress favors apoptosis. ${ }^{37}$ Increased apoptosis has been found in the aneurysm wall of ruptured compared to unruptured aneurysms undergoing treatment. ${ }^{34}$

Additionally, low WSS promotes disorganization of the endothelium and changes the secretion pattern of ECs, leading to increased production of vasoconstrictive and inflammatory agents with decreased vasodilators and antioxidant agents. ${ }^{4}$ Turjman et al. demonstrated that laminar flow promoted ECs to align with the flow and inhibited thrombosis and leukocyte adhesion; turbulent flow caused reorganization of the cells to cuboidal shape and promoted thrombosis and leukocyte adhesion via changes in the cell's metabolism. ${ }^{43}$ Low WSS also promotes selectin-mediated leukocyte rolling, ${ }^{46}$ with lymphocytes having been found to be important in the formation and rupture of aneurysms. ${ }^{36}$ T-lymphocytes promote inflammation in atherosclerotic plaques via secretion of TNF and IFN- $\gamma$, which activates macrophages, B-lymphocytes and increases adhesion molecules. B-lymphocytes modulate the uptake of oxidized lipids by macrophages and can contribute to endothelial damage. According to the work of Sawyer et al., lymphocyte-deficient mice (RAG-1) had significantly fewer intracranial aneurysms compared to wild-type mice at 2 weeks following intracranial aneurysm induction protocol (involving elastase and induced hypertension). ${ }^{36}$ Compared to the wild-type mice, the RAG-1 mice had a lower increase in MMP-2 and MMP-9; additionally, RAG-1 mice had fewer ruptures than wild-type mice. Of note in this study, intracranial aneurysm induction did not alter IFN- $\gamma$, and TNF- $\alpha$ was upregulated similarly in both groups, suggesting an inflammatory pathway independent 
TABLE 1. Summary of hemodynamic pattern and vascular wall changes

\begin{tabular}{|c|c|c|}
\hline Authors \& Year & Hemodynamic Pattern & Vascular Wall Changes \\
\hline Boussel et al., 2008 & \multirow[t]{4}{*}{ Low WSS } & $\begin{array}{l}\text { Disorganization of endothelium, increased production of vasoconstrictive \& inflammatory agents; } \\
\text { increased apoptosis of ECs }\end{array}$ \\
\hline Lu \& Kassab, 2011 & & Decreased production of PGI2, leading to atherosclerotic changes; increased oxidative stress \\
\hline Zhou et al., 2017 & & Increased selectin-mediated leukocyte rolling \\
\hline Papadaki et al., 1998 & & Decreased tPA production \\
\hline Hoi et al., 2008 & \multirow[t]{6}{*}{ High WSS } & MMP production by ECs \\
\hline Fukuda \& Aoki, 2015 & & NF-kB \& COX-2 increased \\
\hline Pawlowska et al., 2018 & & IL-1 $\beta$ increased \\
\hline Taylor et al., 2015 & & $\begin{array}{l}\text { Migration of SMC from media to intima; change of SMC phenotype from contractile to secretory, w/ } \\
\text { MMP production by SMC }\end{array}$ \\
\hline Shi \& Tarbell, 2011 & & PDGF \& FGF-2 released from SMCs \\
\hline Papadaki et al., 1998 & & Increased tPA production \\
\hline \multirow[t]{2}{*}{ Dolan et al., $2013^{10}$} & Negative WSSG & Counteracted upregulation of genes that would be normally upregulated in regions of WSS \\
\hline & Positive WSSG & $\begin{array}{l}\text { Downregulated genes that inhibit progression of cell cycle \& proliferation; decreased expression of } \\
\text { anti-apoptotic genes; downregulated genes associated w/ inflammation \& chemotaxis; increased } \\
\text { ADAMTS1 (ECM protease) \& decreased TAGLN (repressor of MMP-9) }\end{array}$ \\
\hline \multirow[t]{2}{*}{ Turjman et al., 2014} & Laminar flow & EC alignment w/ flow; inhibition of thrombosis \& leukocyte adhesion \\
\hline & Turbulent flow & Reorganized ECs w/ cuboidal shape; promoted thrombosis \& leukocyte adhesion \\
\hline
\end{tabular}

of lymphocytes. ${ }^{36} \mathrm{~T}$ cells, while found in aneurysm walls, were found to be unnecessary for macrophage-mediated degenerative changes according to Miyata et al. ${ }^{29}$

Chronic high WSS, as perceived by molecular sensors such as integrins and/or mechanosensitive ion channels on ECs, causes matrix metalloproteinase (MMP) production that leads to ECM degeneration in the vessel wall, which allows for luminal expansion, as well as increased endothelial growth factor production. ${ }^{18}$ Degradation of the IEL then allows proliferating cells to migrate into the new/enlarged area. As the diameter of the vessel increases, WSS is reduced and matrix degradation stimuli are also decreased..$^{18}$

ECs also interact with SMCs via production of nitric oxide (NO) and prostaglandin (specifically, $\mathrm{PGI}_{2}$, which acts as a vasodilator). ${ }^{26} \mathrm{NO}$ helps to regulate vascular tone ${ }^{25,35}$ and it decreases expression of MCP-1 and VCAM1 , which assists in leukocyte adhesion. ${ }^{38}$ WSS, sensed by EC surface molecules, induces vasodilation via increased NO production. ${ }^{26}$ As ECs continue to be damaged, there is decreased NO synthase in response to elevated WSS.,24 Under conditions of low WSS, there is decreased production of $\mathrm{PGI}_{2}$, contributing to atherosclerotic changes in the aneurysm, as well as increased oxidative stress due to increased oxidase activity and decreased superoxide scavenger. ${ }^{26}$ Increased atherosclerosis within the aneurysm wall has been correlated with aneurysm growth ${ }^{23}$ and wall degeneration. ${ }^{14,31}$

ECs, as discussed in the preceding paragraph, demonstrate increased expression of MCP-1 as well as VCAM1 when damaged. The increase in MCP-1 and VCAM-1 likely occurs via activation of the transcription factor $\mathrm{NF}-x \beta .{ }^{42} \mathrm{NF}-x \beta$ activation occurs via IL- $1 \beta$ and the TNF family of ligands and leads to expression of several proinflammatory proteins (including COX-2 and prostaglandin E2) and proteases, ${ }^{33}$ as well as regulating macrophage ac- tivation and recruitment. ${ }^{7}$ According to the work by Aoki et al., ${ }^{3}$ there exists a positive feedback loop of the COX2/ prostaglandin E2/prostaglandin E2 receptor pathway and $\mathrm{NF}-x \beta$ signaling pathway, which is initiated under high WSS in ECs. Additionally, they found that EP2 deficiency or administration of a COX-2 inhibitor suppressed aneurysm formation and the inflammatory response in arterial walls. ${ }^{3,15}$ Aspirin, an inhibitor of COX-2, has been found to decrease the incidence of cerebral aneurysm rupture, ${ }^{17}$ however, further studies may be needed to determine if COX-2 inhibition is the protective factor associated with aspirin use.

Not only is the EC sensitive to WSS, it is also affected by the WSSFG. ${ }^{10}$ According to the work by Dolan et al., positive WSSG downregulates genes that inhibit progression of the cell cycle and proliferation, decreased expression of genes that inhibited apoptosis, and downregulated genes associated with inflammation (such as those related to immune response and chemotaxis). Additionally, positive WSSG caused higher expression of ADAMTS1 (an ECM protease known to degrade ECM proteins, including aggrecan, versican, and thrombospondin), and downregulated TAGLN (a repressor of collagenase [MMP-9] expression). Also demonstrated in their study, a negative WSSG (even in areas of high WSS) counteracted the upregulation of genes that would have been upregulated by higher WSS. With an increase in cell turnover combined with increased apoptosis and ECM degradation under conditions of positive WSSG, the arterial wall may be more vulnerable to further mechanical damage., 910

The IEL separates the intima (the monolayer of ECs on the luminal side in direct contact with blood flow) from the media (the concentric SMCs and type III collagen). Liaw et al. demonstrated that IEL loss following endothelial injury is due to increased MMP-2 and -9 production by the $\mathrm{SMCs} .{ }^{24}$ 

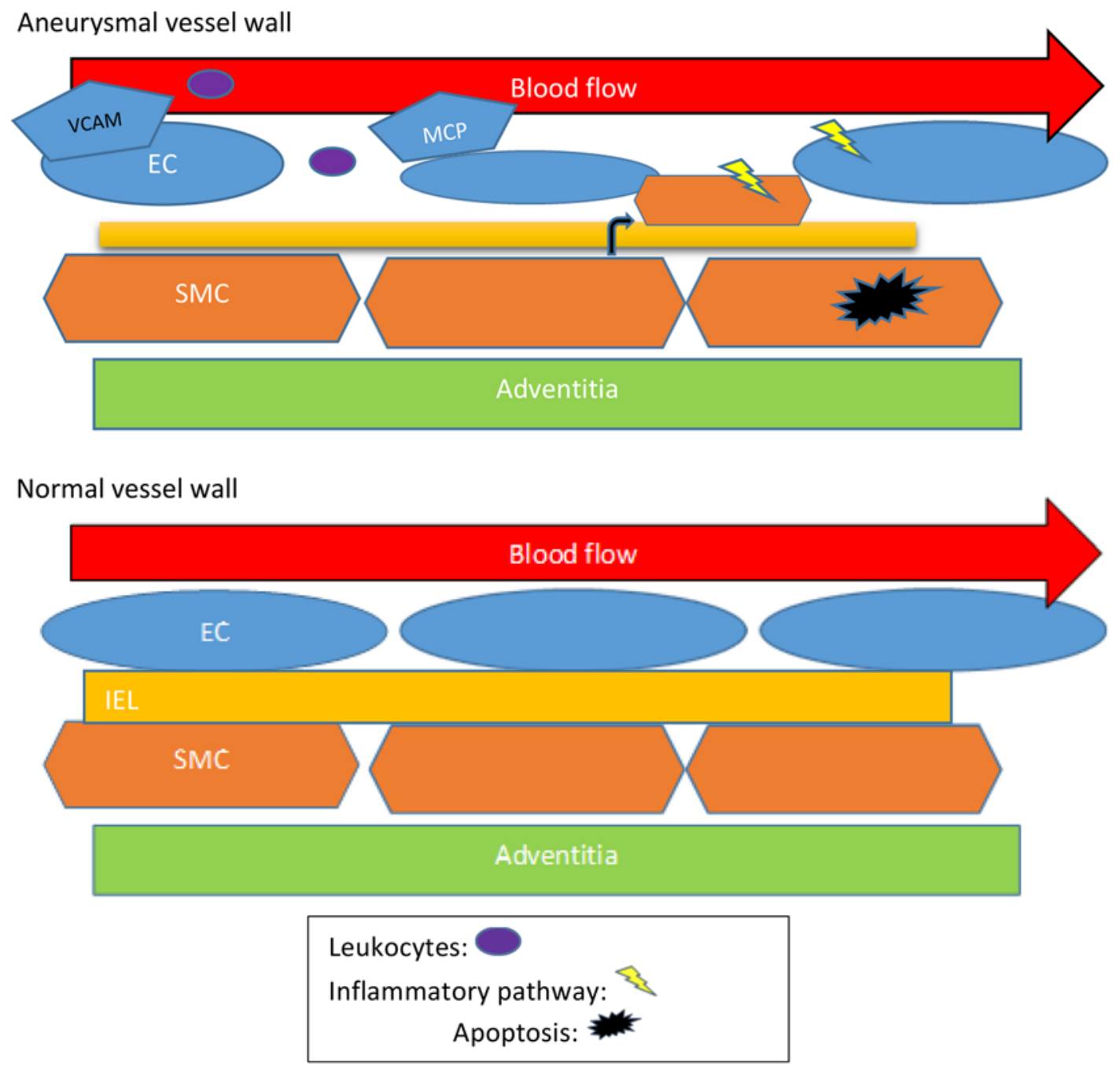

FIG. 1. Vessel wall structure under normal (lower) and aneurysmal (upper) conditions. As discussed in text, vessel wall structure changes induced by WSS include increased production of chemokines and attachment molecules (MCPs and VCAMs) for leukocytes (purple oval), induction of inflammatory pathways in both ECs and SMCs (yellow bolt). The EC layer (blue oval) becomes disorganized with decreased ECM and thinning of the underlying IEL (yellow bar). Additionally, there is migration of the SMCs (orange hexagon) of the media into the intima, with phenotypic SMC changes from contractile to secretory function followed by increased apoptosis (black star) of the SMCs.

\section{Shear Stress and SMC Phenotype Changes}

SMCs are normally not exposed to WSS unless the endothelial layer is damaged; they do, however, react to increased pressure and wall tension by contraction, change of phenotype, proliferation, or synthesis of new matrix. ${ }^{37}$ Arterial SMCs can undergo changes in phenotype, changing from the contraction-oriented phenotype to a proinflammatory/matrix remodeling phenotype. ${ }^{11,16}$

SMCs initially respond to increased WSS by migrating from the tunica media to the intima and, in response to local signals, change from a contractile to a secretory phenotype with increased production of NF- $x \beta$, IL-1 $1 \beta$, TNF- $\alpha$, MMPs, and initiation of apoptotic pathways. ${ }^{40}$ Because SMCs are largely responsible for production of the surrounding ECM, their loss results in loss of this tissue layer. ${ }^{40} \mathrm{IL}-1 \beta$ is induced in the early stages of an- eurysm formation in mice and localizes to the SMC, its activity promotes apoptosis of SMC. IL-1 $\beta$ knockout mice had no significant difference in the rate of aneurysm formation compared to control mice, but they did have fewer advanced aneurysms and lower rates of apoptosis identified in the aneurysm domes, suggesting IL-1 $\beta$ may be important in the progression of aneurysms.

Macrophages have been found to produce MMP-2 and MMP-9, which contribute to the degradation of the ECM. ${ }^{1}$ In a study by Mandelbaum et al., SMCs were also found to have proinflammatory responses to aneurysm-inducing hemodynamic changes. Aneurysm damage was induced via bilateral common carotid artery ligation in rabbits, and it was found that MMP-2 and MMP-9 increased in areas of aneurysm damage within 5 days following flow-increasing surgery. Inflammatory cells in the areas of dam- 
age were sparse, and the MMPs colocalized with SMCs, suggesting that SMCs are also producers of MMPs. Additionally, in this study, macrophage depletion did not decrease the initiation of aneurysm changes. There was a change from the normal contractile phenotype of SMCs in areas of flow-induced damage, with decreased expression of SMA (a marker of smooth muscle) and calponin, as well as increased expression of inflammatory markers (NF- $x \mathrm{~B}$ and MCP-1). ${ }^{27}$

When exposed to shear stress, PDGF and FGF-2 are released from SMCs. ${ }^{37}$ PDGF influences SMC migration and cell adhesion, decreases SMA expression, and results in less dense ECM structure. ${ }^{39}$ Frösen et al. demonstrated that receptors for TGF- $\beta$, PDGF, and VEGF were upregulated in ruptured aneurysms and were associated with aneurysm wall remodeling; warning leaks were associated with increased GFG receptor expression. ${ }^{12}$ As discussed in a review by Signorelli and colleagues, SMC phenotype modulation is affected by FGF and TGF- $\beta .^{38}$ SMCs also have been found to have Ets-1 transcription factor expression after experimentally induced aneurysm induction; Ets-1 mediates expression of MCP-1, which attracts monocytes and macrophages. ${ }^{2}$

Production of tissue plasminogen activator (tPA) and human protease activated receptor -1 have been found to increase under high WSS and decrease under low WSS. ${ }^{32}$ These findings are interesting to note with consideration of Frösen and colleagues' findings of luminal thrombosis to be found more frequently in ruptured than nonruptured cerebral aneurysms.$^{13}$ Additionally, Frösen et al. demonstrated that vessel walls with linearly organized SMCs had a lower rate of rupture than those with disorganized SMCs; shear stress has been found to influence the alignment of SMCs with differences in alignment based on uniform and nonuniform shear stress. ${ }^{37}$

\section{Conclusions}

Changes in the aneurysm wall leading to aneurysm progression and rupture are mediated by cellular changes induced by both high and low shear stress. Readers are directed to Table 1 for a summary of vascular wall changes based on patterns of shear stress and Fig. 1, which further illustrates how the different modes of shear stress contribute to aneurysm changes in the vascular wall. Low shear stress conditions favor disorganization and apoptosis of the EC, as well as oxidative stress and additional inflammation. High shear stress promotes the migration of SMCs and phenotypic changes leading to SMC secretion of inflammatory mediators and factors involved in the degradation of the vessel wall. Inflammatory signaling occurs not only from leukocytes responding to cytokines produced by EC but also from the vascular SMCs, which have undergone a phenotypic change.

Understanding the vessel wall's response to WSS is fundamental to appreciating the sequence of events leading to aneurysm formation and growth, thereby providing potential areas in which we may influence the natural history of aneurysms so as to prevent rupture and the neurological morbidity that ensues. Previous work suggests that medications, such as aspirin and selective COX-2 in- hibitors, potentially decrease the risk of aneurysm rupture. Moving forward, with a greater understanding of aneurysm wall changes, it may be possible to selectively target and perhaps even reverse the pathological process leading to aneurysm growth and rupture.

\section{References}

1. Aoki T, Kataoka H, Morimoto M, Nozaki K, Hashimoto N: Macrophage-derived matrix metalloproteinase-2 and -9 promote the progression of cerebral aneurysms in rats. Stroke 38:162-169, 2007

2. Aoki T, Kataoka H, Nishimura M, Ishibashi R, Morishita $\mathrm{R}$, Miyamoto S: Ets-1 promotes the progression of cerebral aneurysm by inducing the expression of MCP-1 in vascular smooth muscle cells. Gene Ther 17:1117-1123, 2010

3. Aoki T, Nishimura M, Matsuoka T, Yamamoto K, Furuyashiki T, Kataoka H, et al: PGE(2) -EP(2) signalling in endothelium is activated by haemodynamic stress and induces cerebral aneurysm through an amplifying loop via NF- $x$ B. Br J Pharmacol 163:1237-1249, 2011

4. Boussel L, Rayz V, McCulloch C, Martin A, Acevedo-Bolton G, Lawton M, et al: Aneurysm growth occurs at region of low wall shear stress: patient-specific correlation of hemodynamics and growth in a longitudinal study. Stroke 39:29973002,2008

5. Cebral JR, Detmer F, Chung BJ, Choque-Velasquez J, Rezai B, Lehto H, et al: Local hemodynamic conditions associated with focal changes in the intracranial aneurysm wall. AJNR Am J Neuroradiol 40:510-516, 2019

6. Cebral JR, Sheridan M, Putman CM: Hemodynamics and bleb formation in intracranial aneurysms. AJNR Am J Neuroradiol 31:304-310, 2010

7. Chalouhi N, Ali MS, Jabbour PM, Tjoumakaris SI, Gonzalez LF, Rosenwasser RH, et al: Biology of intracranial aneurysms: role of inflammation. J Cereb Blood Flow Metab 32:1659-1676, 2012

8. Doddasomayajula R, Chung BJ, Mut F, Jimenez CM, Hamzei-Sichani F, Putman CM, et al: Hemodynamic characteristics of ruptured and unruptured multiple aneurysms at mirror and ipsilateral locations. AJNR Am J Neuroradiol 38:2301-2307, 2017

9. Dolan JM, Kolega J, Meng H: High wall shear stress and spatial gradients in vascular pathology: a review. Ann Biomed Eng 41:1411-1427, 2013

10. Dolan JM, Meng H, Sim FJ, Kolega J: Differential gene expression by endothelial cells under positive and negative streamwise gradients of high wall shear stress. Am J Physiol Cell Physiol 305:C854-C866, 2013

11. Frösen J: Smooth muscle cells and the formation, degeneration, and rupture of saccular intracranial aneurysm wall-a review of current pathophysiological knowledge. Transl Stroke Res 5:347-356, 2014

12. Frösen J, Piippo A, Paetau A, Kangasniemi M, Niemelä M, Hernesniemi J, et al: Growth factor receptor expression and remodeling of saccular cerebral artery aneurysm walls: implications for biological therapy preventing rupture. Neurosurgery 58:534-541, 2006

13. Frösen J, Piippo A, Paetau A, Kangasniemi M, Niemelä M, Hernesniemi J, et al: Remodeling of saccular cerebral artery aneurysm wall is associated with rupture: histological analysis of 24 unruptured and 42 ruptured cases. Stroke 35:2287-2293, 2004

14. Frösen J, Tulamo R, Heikura T, Sammalkorpi S, Niemelä M, Hernesniemi J, et al: Lipid accumulation, lipid oxidation, and low plasma levels of acquired antibodies against oxidized lipids associate with degeneration and rupture of the intracranial aneurysm wall. Acta Neuropathol Commun 1:71, 2013 
15. Fukuda M, Aoki T: Molecular basis for intracranial aneurysm formation. Acta Neurochir Suppl (Wien) 120:13-15, 2015

16. Hao H, Gabbiani G, Bochaton-Piallat ML: Arterial smooth muscle cell heterogeneity: implications for atherosclerosis and restenosis development. Arterioscler Thromb Vasc Biol 23:1510-1520, 2003

17. Hasan DM, Mahaney KB, Brown RD Jr, Meissner I, Piepgras DG, Huston J, et al: Aspirin as a promising agent for decreasing incidence of cerebral aneurysm rupture. Stroke 42:3156-3162, 2011

18. Hoi Y, Gao L, Tremmel M, Paluch RA, Siddiqui AH, Meng $\mathrm{H}$, et al: In vivo assessment of rapid cerebrovascular morphological adaptation following acute blood flow increase. J Neurosurg 109:1141-1147, 2008

19. Jou LD, Lee DH, Morsi H, Mawad ME: Wall shear stress on ruptured and unruptured intracranial aneurysms at the internal carotid artery. AJNR Am J Neuroradiol 29:1761-1767, 2008

20. Jung KH: New pathophysiological considerations on cerebral aneurysms. Neurointervention 13:73-83, 2018

21. Kadasi LM, Dent WC, Malek AM: Colocalization of thinwalled dome regions with low hemodynamic wall shear stress in unruptured cerebral aneurysms. J Neurosurg 119:172-179, 2013

22. Kataoka K, Taneda M, Asai T, Kinoshita A, Ito M, Kuroda R: Structural fragility and inflammatory response of ruptured cerebral aneurysms. A comparative study between ruptured and unruptured cerebral aneurysms. Stroke 30:1396-1401, 1999

23. Kosierkiewicz TA, Factor SM, Dickson DW: Immunocytochemical studies of atherosclerotic lesions of cerebral berry aneurysms. J Neuropathol Exp Neurol 53:399-406, 1994

24. Liaw N, Fox JM, Siddiqui AH, Meng H, Kolega J: Endothelial nitric oxide synthase and superoxide mediate hemodynamic initiation of intracranial aneurysms. PLoS One 9:e101721, 2014

25. Loufrani L, Henrion D: Role of the cytoskeleton in flow (shear stress)-induced dilation and remodeling in resistance arteries. Med Biol Eng Comput 46:451-460, 2008

26. Lu D, Kassab GS: Role of shear stress and stretch in vascular mechanobiology. J R Soc Interface 8:1379-1385, 2011

27. Mandelbaum M, Kolega J, Dolan JM, Siddiqui AH, Meng H: A critical role for proinflammatory behavior of smooth muscle cells in hemodynamic initiation of intracranial aneurysm. PLoS One 8:e74357, 2013

28. Meng H, Tutino VM, Xiang J, Siddiqui A: High WSS or low WSS? Complex interactions of hemodynamics with intracranial aneurysm initiation, growth, and rupture: toward a unifying hypothesis. AJNR Am J Neuroradiol 35:1254-1262, 2014

29. Miyata H, Koseki H, Takizawa K, Kasuya H, Nozaki K, Narumiya $S$, et al: T cell function is dispensable for intracranial aneurysm formation and progression. PLoS One 12:e0175421, 2017

30. Munarriz PM, Gómez PA, Paredes I, Castaño-Leon AM, Cepeda S, Lagares A: Basic principles of hemodynamics and cerebral aneurysms. World Neurosurg 88:311-319, 2016

31. Ollikainen E, Tulamo R, Lehti S, Lee-Rueckert M, Hernesniemi J, Niemelä M, et al: Smooth muscle cell foam cell formation, apolipoproteins, and ABCA1 in intracranial aneurysms: Implications for lipid accumulation as a promoter of aneurysm wall rupture. J Neuropathol Exp Neurol 75:689-699, 2016

32. Papadaki M, Ruef J, Nguyen KT, Li F, Patterson C, Eskin SG, et al: Differential regulation of protease activated receptor-1 and tissue plasminogen activator expression by shear stress in vascular smooth muscle cells. Circ Res 83:1027-1034, 1998
33. Pawlowska E, Szczepanska J, Wisniewski K, Tokarz P, Jaskólski DJ, Blasiak J: NF- $x$ B-mediated inflammation in the pathogenesis of intracranial aneurysm and subarachnoid hemorrhage. Does autophagy play a role? Int J Mol Sci 19:E1245, 2018

34. Pentimalli L, Modesti A, Vignati A, Marchese E, Albanese A, Di Rocco F, et al: Role of apoptosis in intracranial aneurysm rupture. J Neurosurg 101:1018-1025, 2004

35. Santiago-Sim TKD: Pathobiology of intracranial aneurysms, in Winn H (ed): Youmans Neurological Surgery, Vol 4, ed 6. Philadelphia: Elsevier, pp 3747-3755, 2011

36. Sawyer DM, Pace LA, Pascale CL, Kutchin AC, O’Neill $\mathrm{BE}$, Starke RM, et al: Lymphocytes influence intracranial aneurysm formation and rupture: role of extracellular matrix remodeling and phenotypic modulation of vascular smooth muscle cells. J Neuroinflammation 13:185, 2016

37. Shi ZD, Tarbell JM: Fluid flow mechanotransduction in vascular smooth muscle cells and fibroblasts. Ann Biomed Eng 39:1608-1619, 2011

38. Signorelli F, Gory B, Riva R, Labeyrie PE, Pelissou-Guyotat I, Turjman F: Hemodynamics, inflammation, vascular remodeling, and the development and rupture of intracranial aneurysms: a review. Neuroimmunol Neuroinflamm 2:59-67, 2015

39. Stegemann JP, Hong H, Nerem RM: Mechanical, biochemical, and extracellular matrix effects on vascular smooth muscle cell phenotype. J Appl Physiol (1985) 98:2321-2327, 2005

40. Taylor BES, Appelboom G, Zilinyi R, Goodman A, Chapel $\mathrm{D}$, LoPresti M, et al: Role of the complement cascade in cerebral aneurysm formation, growth, and rupture. Neuroimmunol Neuroinflamm 2:93-101, 2015

41. Texakalidis P, Hilditch CA, Lehman V, Lanzino G, Pereira VM, Brinjikji W: Vessel wall imaging of intracranial aneurysms: systematic review and meta-analysis. World Neurosurg 117:453-458, 458.e1, 2018

42. Tulamo R, Frösen J, Hernesniemi J, Niemelä M: Inflammatory changes in the aneurysm wall: a review. J Neurointerv Surg 10 (Suppl 1):i58-i67, 2018

43. Turjman AS, Turjman F, Edelman ER: Role of fluid dynamics and inflammation in intracranial aneurysm formation. Circulation 129:373-382, 2014

44. Turkmani AH, Edwards NJ, Chen PR: The role of inflammation in cerebral aneurysms. Neuroimmunol Neuroinflamm 2:102-106, 2015

45. Xiang J, Natarajan SK, Tremmel M, Ma D, Mocco J, Hopkins LN, et al: Hemodynamic-morphologic discriminants for intracranial aneurysm rupture. Stroke 42:144-152, 2011

46. Zhou G, Zhu Y, Yin Y, Su M, Li M: Association of wall shear stress with intracranial aneurysm rupture: systematic review and meta-analysis. Sci Rep 7:5331, 2017

\section{Disclosures}

Dr. Prestigiacomo is a consultant for Aesculap and Thermopeutix. He serves as a member of the board with IBRF and Cerenovus.

\section{Author Contributions}

Conception and design: Staarmann. Drafting the article: Staarmann. Critically revising the article: Smith, Prestigiacomo. Reviewed submitted version of manuscript: Smith, Prestigiacomo. Study supervision: Prestigiacomo.

\section{Correspondence}

Brittany Staarmann: University of Cincinnati Medical Center, Cincinnati, OH. staarmby@mail.uc.edu. 